\title{
Article \\ Stimulus-Responsive Shrinkage in Electrospun Membranes: Fundamentals and Control
}

\author{
Feiyu Fang ${ }^{1,2}$, Han Wang ${ }^{1,2, *}$, Huaquan Wang ${ }^{3}$, Wei Min Huang ${ }^{4, * \mathbb{D}}$, Yahui Chen ${ }^{5}$, Nian Cai ${ }^{6}$, Xindu Chen ${ }^{1,2}$ \\ and Xin Chen ${ }^{1,2}$
}

check for updates

Citation: Fang, F.; Wang, H.; Wang, H.; Huang, W.M.; Chen, Y.; Cai, N.; Chen, $X$.; Chen, X. Stimulus-Responsive Shrinkage in Electrospun Membranes: Fundamentals and Control. Micromachines 2021, 12, 920. https:// doi.org/10.3390/mi12080920

Academic Editor: Dong-Sung Kim

Received: 8 July 2021

Accepted: 30 July 2021

Published: 31 July 2021

Publisher's Note: MDPI stays neutral with regard to jurisdictional claims in published maps and institutional affiliations.

Copyright: (C) 2021 by the authors Licensee MDPI, Basel, Switzerland. This article is an open access article distributed under the terms and conditions of the Creative Commons Attribution (CC BY) license (https:// creativecommons.org/licenses/by/ $4.0 /)$.
1 Guangdong Provincial Key Laboratory of Micro-Nano Manufacturing Technology and Equipment, School of Electromechanical Engineering, Guangdong University of Technology, Guangzhou 510006, China; feiyu93@foxmail.com (F.F.); chenxindu@gdut.edu.cn (X.C.); chenx@gdut.edu.cn (X.C.)

2 State Key Laboratory of Precision Electronic Manufacturing Technology and Equipment, School of Electromechanical Engineering, Guangdong University of Technology, Guangzhou 510006, China

3 China Resources Cement Technology Research and Development Co., Ltd., Guangzhou 510460, China; whq931@163.com

4 School of Mechanical and Aerospace Engineering, Nanyang Technological University, 50 Nanyang Avenue, Singapore 639798, Singapore

5 School of Physical Science and Technology, Soochow University, Suzhou 215006, China; chenyahui@suda.edu.cn

6 School of Information Engineering, Guangdong University of Technology, Guangzhou 510006, China; cainian@gdut.edu.cn

* Correspondence: wanghangood@gdut.edu.cn (H.W.); mwmhuang@ntu.edu.sg (W.M.H.)

Abstract: Shrinkage is observed in many electrospun membranes. The stretched conformation of the macromolecular chains has been proposed as the possible cause. However, so far, our understanding of the fundamentals is still qualitative and cannot provide much help in the shrinkage control. In this paper, based on the crimped fibers after stimulus-induced shrinkage, a clear evidence of buckling, the gradient pre-strain field in the cross-section of the electrospun fibers, which is the result of a gradient solidification field and a tensile force in the fibers during electrospinning, is identified as the underlying mechanism for the stimulus-induced shrinkage. Subsequently, two buckling conditions are derived. Subsequently, a series of experiments are carried out to reveal the influence of four typical processing parameters (namely, the applied voltage, solution concentration, distance between electrodes, and rotation speed of collector), which are highly relevant to the formation of the gradient pre-strain field. It is concluded that there are some different ways to achieve the required shrinkage ratios in two in-plane directions (i.e., the rotational and transverse directions of the roller collector). Some of the combinations of these parameters are more effective at achieving high uniformity than others. Hence, it is possible to optimize the processing parameters to produce high-quality membranes with well-controlled shrinkage in both in-plane directions.

Keywords: electrospinning; shrinkage; buckling; shape memory effect; gradient strain field

\section{Introduction}

Electrospinning is a commonly used technology for producing fiber/nanofiber membranes and has been widely used in tissue engineering, filtration systems, flexible electronics, etc. [1-6]. Many electrospinning fiber membranes (e.g., polyvinyl alcohol (PVA), poly (lactic acid) (PLA), poly(lactide-co-glycolide) (PLGA), polysulfone (PSF), polyvinylpyrrolidone (PVP), poly- $\varepsilon$-caprolactone (PCL)) shrink under certain conditions, e.g., upon heating and/or immersing in a right solvent [4,5,7-12]. Such a kind of shrinkage may cause some serious problems (such as scaffold deformation, collapse, etc.) in tissue engineering applications, but they also can be utilized, e.g., to prepare crimped fibers and self-folding electrospun scaffolds $[4,5,9,13]$. Although crimped fibers can be produced via co-electrospinning of two different materials with different shrinkage ratios [13,14], in addition to a far more sophisticated system, a special nozzle is required [15]. 
The stretched conformation of the macromolecular chains has been suggested as the cause of the shrinkage of the electrospun membranes [4,5]. The network of entangled polymer chains of semidilute entangled solution is stretched by the external force. It leads to a steady high molecular orientation and is frozen due to the rapid evaporation of the solvent. This unbalanced molecular conformation recovers under the right external stimuli, so the fibers shrink. However, at present, this explanation is only at a qualitative level and cannot provide much useful guidance for controlling the shrinkage, either to avoid undesired shape change or to be harnessed in a precise manner for real engineering applications.

The shape memory effect (SME) refers to the ability of a material to recover its original shape, but only when the right stimulus is applied. Those materials with the SME are called shape memory materials (SMMs) $[16,17]$. Depending on the type of SMM, the stimuli that can active the SME include heat (thermo-responsive), chemical (including water, chemoresponsive), light (photo-responsive) and magnetic field (magneto-responsive). While the SME is limited to some particular alloys (called shape memory alloys, SMAs), most polymers intrinsically have the heating-responsive SME and chemo-responsive SME [18]. For a lot of engineering polymers, some chemicals (including water and even moisture) may act as a plasticizer to reduce their glass transition temperature $\left(T_{g}\right)$ even below the room temperature [19]. Consequently, their shape recovery can be activated by immersing in the right chemical without the heating process [20]. In addition to the plasticizing effect, which is generic for the glass transition-based SME in polymers, some other working mechanisms are able to induce the chemo-responsive SME in some special types of polymers [21,22].

A typical shape memory cycle includes two processes [23]. The first process (called programming) is to fix the temporary shape, and the second process (called recovery) is to apply the right stimulus to active the SME. The programming process is required to store the elastic energy in the elastic part of SMPs [18]. The stored elastic energy provides the driving force for shape recovery in the step. The programming process may be integrated in the material fabrication/processing part [24].

The purpose of this paper is twofold. One is to reveal the fundamentals behind the shrinkage of the electrospun membranes, including the underlying mechanism and the critical buckling conditions. The other is to investigate the feasibility of controlling the shrinkage in two in-plane directions of the electrospun membranes via a series of experiments, in which four key processing parameters, identified based on the understanding of the fundamentals, are chosen as the variables.

\section{Materials and Method}

\subsection{Materials and Preparation}

The thermoplastic polyurethane (TPU) MM5520 (pellets) used for electrospinning was purchased from SMP Technologies Inc. (Tokyo, Japan), with a nominal glass transition temperature $\left(\mathrm{T}_{\mathrm{g}}\right)$ of $55^{\circ} \mathrm{C}$. As reported in [25], after being immersed in water or ethanol, the $\mathrm{T}_{\mathrm{g}}$ of this TPU can be lowered by $30^{\circ} \mathrm{C}$, due to the plasticizing effect of the absorbed bound water or ethanol on the hydrogen bonding [26]. Hence, the SME of this TPU can be activated either by heating to above its $\mathrm{T}_{\mathrm{g}}$ or by wetting in ethanol.

$\mathrm{N}, \mathrm{N}$-dimethylformamide (DMF), ethanol, and acetone were purchased from Sinopharm Chemical Reagent Co., Ltd. (Shanghai, China). DMF was mixed with acetone in a volumetric ratio of 1:1. After that, TPU pellets were added into it for different concentrations varying from $10 \mathrm{wt} \%$ to $25 \mathrm{wt} \%$. Subsequently, magnetic stirring was applied at room temperature $\left(\approx 25^{\circ} \mathrm{C}\right)$ for $24 \mathrm{~h}$.

\subsection{Fabrication of Electrospun Membranes}

Figure 1 schematically illustrates the electrospinning system used in the course of this study. The prepared solution for electrospinning was placed into a syringe equipped with a $22 \mathrm{G}$ dispensing nozzle (inner diameter $420 \mu \mathrm{m}$ and external diameter $720 \mu \mathrm{m}$ ), and a pump (Pump 11 Elite, Harvard Inc., Holliston, MA, USA) was used to feed $5 \mathrm{~mL}$ of solution at a constant speed for membrane fabrication. A high-voltage supply (DW-P303, Tianjin 
Dongwen Inc., Tianjin, China) was applied to the nozzle. A speed-adjustable rotating cylinder (with a diameter of $10 \mathrm{~cm}$ covered by aluminum foil and width of $5 \mathrm{~cm}$ ) served as the collector and was connected to the negative electrode. The applied voltage $(\mathrm{kV})$, solution concentration ( $\mathrm{wt} \%$ ), distance between electrodes $(\mathrm{cm})$, rotation speed of the collector $(\mathrm{rpm} / \mathrm{min})$, and solution flow rate $(\mathrm{mL} / \mathrm{h})$ were taken as the control parameters in the fabrication process. The electrospinning experiments were performed at room temperature without any special control in humidity. Refer to Table S1 in the Supplementary Materials for the processing parameters of each group.

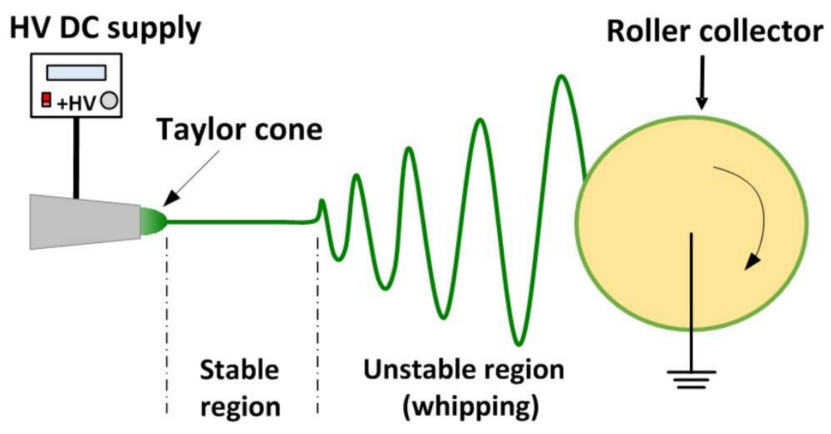

Figure 1. Schematic illustration of electrospinning system.

\subsection{Shrinkage Test}

As-fabricated TPU membranes were taken out of the roller collector. Small samples with a size of about $4.8 \mathrm{~cm} \times 4.8 \mathrm{~cm}$ were cut from the membranes. It is noticed that when the membranes were taken out of the roller collector, about $4 \%$ shrinkage in both the rotational direction and the transverse direction was observed. Ethanol (concentration: 99\%) was used to activate the shrinkage of TPU samples. To avoid tangling due to rapid and severe shrinking, TPU samples were wetting by ethanol droplets from one side to the other side gradually. Refer to Video S1 in Supplementary Materials for a video of the process of the controlled ethanol wetting of membrane. TPU samples were fully wetted for two to three min, until no further deformation was observed. The change in length $\left(\mathrm{D}_{\mathrm{i}}\right.$, $\mathrm{i}=\mathrm{x}$ and $\mathrm{y}$, where $\mathrm{x}$ is the rotational direction of the roller and $\mathrm{y}$ is the transverse direction, respectively) was recorded (Figure $2 \mathrm{a}$ ), and the shrinkage ratios in both the rotational direction of collector and the transverse direction were calculated as $\mathrm{D}_{\mathrm{i}} / \mathrm{L}_{\mathrm{i}, 0}(\%)$, where $\mathrm{L}_{\mathrm{i}, 0}$ is the original length before wetting.
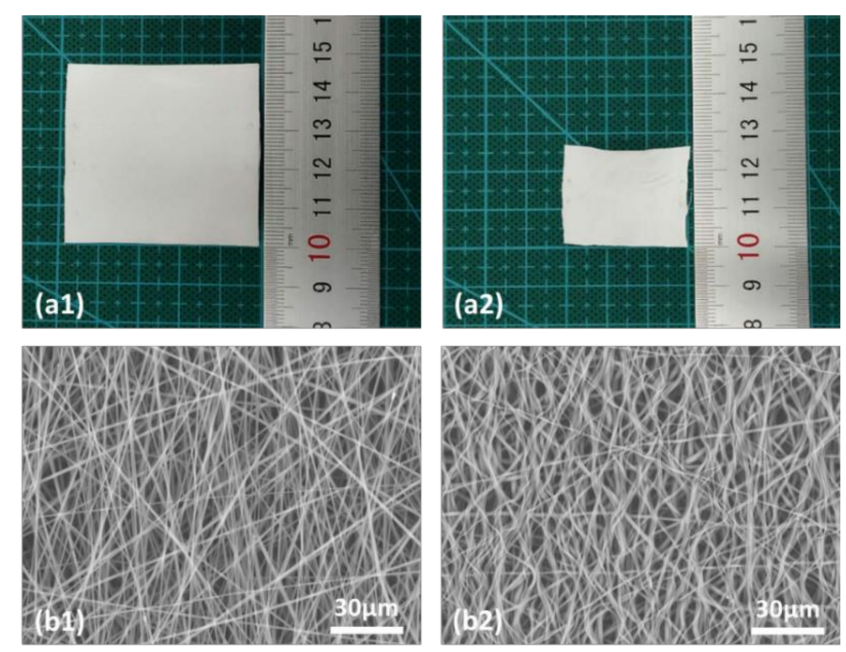

Figure 2. (a) Typical sample (applied voltage: $10 \mathrm{kV}$; solution concentration: $15 \%$; distance between electrodes: $10 \mathrm{~cm}$; speed of collector: $700 \mathrm{rpm} / \mathrm{min}$; solution flow rate: $1.2 \mathrm{~mL} / \mathrm{h}$ ) before (a1) and after (a2) ethanol wetting. (b) SEM images before (b1) and after (b2) wetting. 


\subsection{Morphology Characterization}

The morphology of the samples before and after ethanol wetting was revealed by a scanning electron microscope (SEM; TM3030, Hitachi Inc., Tokyo, Japan). The distribution of the fiber alignment of the as-fabricated membrane was calculated using Image J software.

\section{Results and Discussion}

\subsection{Membrane Shrinkage and Fiber Crimp}

Figure $2 \mathrm{~b}$ presents typical SEM images before and after ethanol wetting. Refer to Figures S1-S3 in Supplementary for more SEM results of this TPU, another TPU, and poly (lactic acid) (PLA), respectively.

The SEM images of the as-fabricated membrane samples and the distribution of the fiber alignment in three samples fabricated at different rotation speeds are presented in Figures S4 and S5 in the Supplementary Materials. It appears that the rotation speed of the roller, in particular a higher rotation speed (e.g., $700 \mathrm{rpm}$ ), does cause more alignment of the fibers, which implies a kind of straightening effect when the fibers are collected on the roller.

About $4 \%$ shrinkage in both directions as we observed during taking the membranes out of the roller does not induce any apparent crimping in the fibers. This finding implies that the shrinkage, which is much smaller when compared with the shrinkage induced by ethanol, is mostly elastic deformation and uniform (i.e., without buckling) in the whole fibers. De-swelling during further evaporation of the solvent after the fibers have been collected by the roller should be the major source for the tensile force, while the straightening effect may have limited the contribution to the tensile force.

\subsection{Underlying Mechanism behind Membrane Shrinkage}

Recall Figure 1. In the electrospinning process, a high voltage is applied on the needle to form a high-voltage electric field. Due to the accumulation of electric charge, the droplet hanging on the needle is stretched into an elongated cone shape, which is known as a Taylor cone [27]. When the voltage reaches a critical value, the electric field causes the charged liquid to overcome the surface tension of the solution and to release an elongated jet from the tip of the cone. Due to the combined action of the electric field force and the Coulomb repulsive force, the charged thin jet becomes unstable and whipping. In the meantime, the diameter of the jet decreases rapidly until the jet becomes fiber and collected by the collector $[15,28]$. If a high-speed rotating drum is used as the collector, a tensile force is applied on the fiber. In this whole process, the solvent evaporates and the liquid jet gradually becomes thin solid fiber.

The evaporation of the solvent is inhomogeneous in the radial direction of the jet/fiber $[29,30]$. As an example, Figure $3 a, b$ reveal that the solidification/drying of the jet starts from the surface of the jet (shell) and gradually moves into the core. The solidified shell (only partially solidified) is stretched in an elastic-plastic manner, while the inner unsolidified core is able to flow easily, which results in a pre-strain in the shell, as illustrated in Figure 3c. Since the solidification/drying process may continue toward the inner core even after the fiber has been collected on the roller collector, while a tensile force is applied, a gradient pre-strain field results in the fibers of the final dry membrane. This is a kind of programming, which stores the elastic energy for later on shape recovery [18,31]. However, since the pre-strain is in a gradient manner within the cross-section, during the ethanol wetting-induced shape recovery process, in which wetting starts from the outer-surface toward the inner part gradually, the pre-strained shell contracts (Figure 3c), while the inner core mostly maintains its shape. The result is buckling/crimping of the fiber, as reported in [25]. If the fiber is about uniformly pre-stretched, buckling may occur, in particular during chemically induced shape recovery [32], but the final shape of the fiber should be more or less straight. 


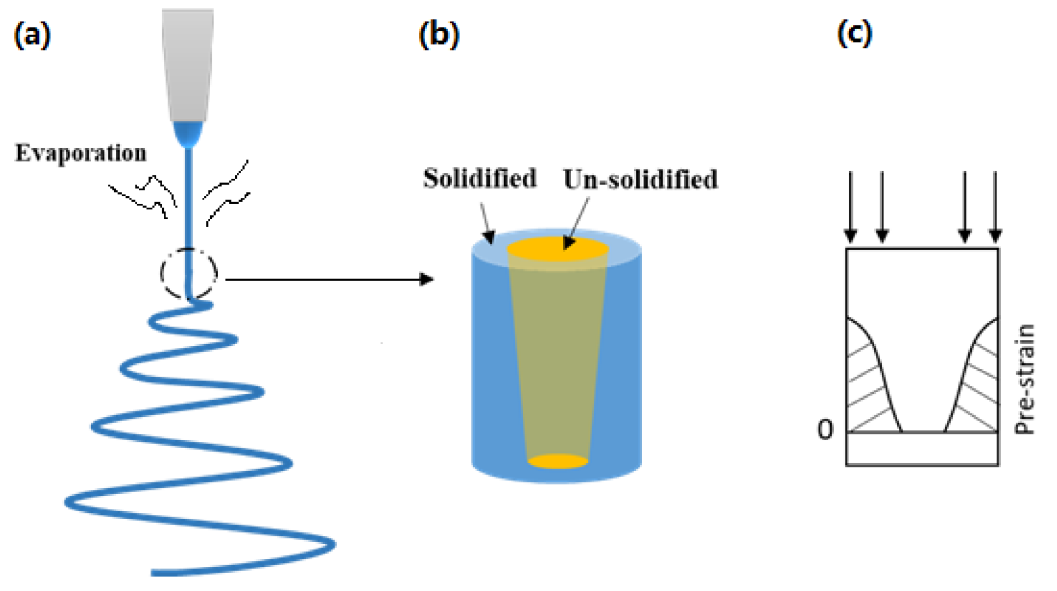

Figure 3. Illustration of electrospinning (a), gradual solidifying (b), and resulted pre-strain field (c).

Refer to Figure 4a for an illustration of the cross-section of the dried fiber (with a radius of $R$ ) and the pre-strain in it. The process of wetting by ethanol should start from the surface of the dry fiber. For simplification, we may assume that $r$ is the radius of the inner core (layer II, without any pre-strain) and the outer shell (layer I) is uniformly pre-strained.

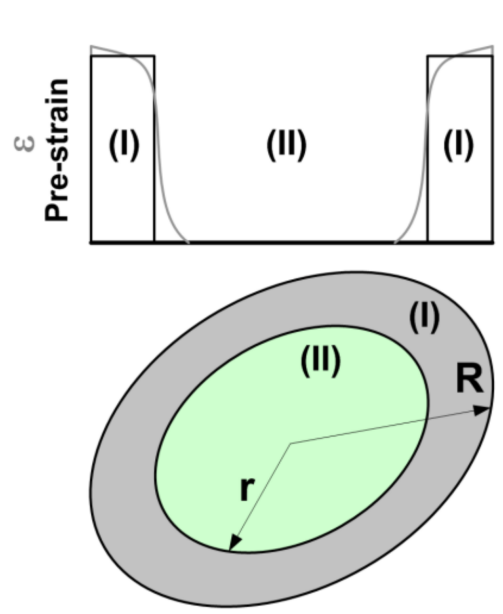

(a)

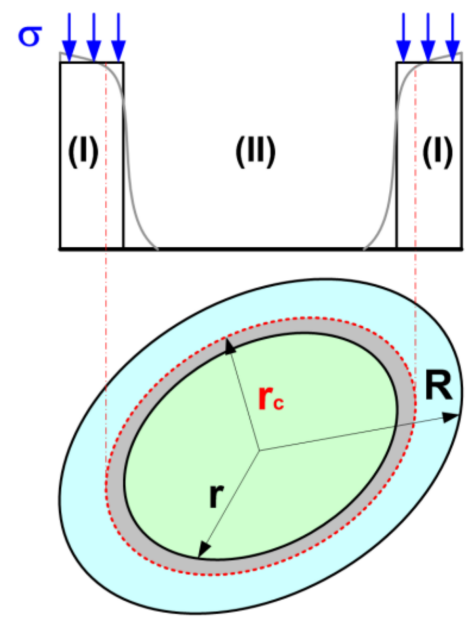

(b)

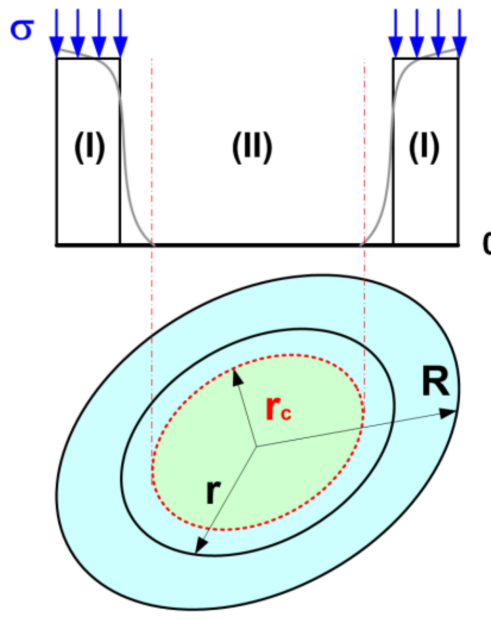

(c)

Figure 4. Illustration of cross-section of dried fiber and pre-strain in it. (a) Before wetting; (b) buckling when the shell is partially wetted; (c) buckling when the core is partially wetted.

In the process of ethanol-induced shape recovery, buckling may occur when the critical ethanol penetration depth $\left(R-r_{c}\right)$ is in the shell $\left(R>r_{c}>r\right)$ (Figure $\left.4 b\right)$ or in the inner core $\left(r>r_{c}\right)$ (Figure $4 \mathrm{c}$ ).

The critical condition for buckling for both cases can be written as [32],

$$
F_{c r}=\frac{\pi^{2} E I}{4 L^{2}}
$$

where $F_{c r}$ is the critical load for buckling; $L$ is the effective length of the fiber, $E I$ is the flexural rigidity.

In the case of $\left(R>r_{c}>r\right)$ (Figure $\left.4 b\right)$,

$$
E I=\frac{\pi}{4}\left[E_{R}\left(R^{4}-r_{c}^{4}\right)+E_{c}\left(r_{c}^{4}-r^{4}\right)+E_{r} r^{4}\right]
$$


where $E_{R}, E_{c}$, and $E_{r}$ are the Young's modulus of the ethanol softened TPU (pre-strained shell), the Young's modulus of the pre-strained but yet softened TPU (shell), and the Young's modulus of the inner core (without pre-strain), respectively.

After wetting by ethanol, the Young's modulus of TPU MM5520 drops dramatically, and the variation in its Young's modulus after pre-stretching is limited [33]. Here, we may define $E_{h}$ and $E_{s}$ as the Young's moduli of TPU MM5520 before and after wetting by ethanol, respectively (i.e., $E_{r}=E_{c}$ ).

Consequently, Equation (2) may be reduced to

$$
E I=\frac{\pi}{4}\left[E_{h} r_{c}^{4}+E_{s}\left(R^{4}-r_{c}^{4}\right)\right] .
$$

The internal compressive force of the wetted/softened part due to the release of elastic energy in the pre-straining part may be estimated by

$$
F=\pi\left(R^{2}-r_{c}^{2}\right) \sigma
$$

where $\sigma$ is the compressive stress acting on the softened part. Same as in [31], we may assume

$$
\sigma=\varepsilon E_{s}
$$

where $\varepsilon$ is the pre-strain.

In the other case of $\left(r \geq r_{c}\right)$ (Figure 4c), the compressive force in the pre-strained shell, i.e.

$$
F=\pi\left(R^{2}-r^{2}\right) \sigma
$$

is not high enough to induce buckling. Partial softening of the inner core is required. Equation (3) is still valid.

Since many fibers with different diameters (the difference might be only slight) and different effective lengths entangle together in a membrane, some fibers may lose their stability earlier than the others during stimulus-activated shape recovery. The buckling condition may also affect the amount of shrinkage of the membrane. However, for a particular polymer, the most important parameters that affect the amount of shrinkage of a membrane should be the effective length of the fibers, the diameter of the fiber, the thickness of the pre-strained shell, the amount of pre-strain in the shell, and the rotation speed of the roller (in the case of 2D shrinkage). Consequently, these processing parameters, such as the applied voltage, solution concentration, distance between electrodes, rotation speed of collector, and solution flow rate, which can affect the solvent evaporation speed, solidification speed, stretching force, and fiber flight time are important factors. The porous ratio, which is indirectly related to the effective length of the fibers, might be important for the critical buckling condition but less influential on the final shrinkage ratio.

\subsection{D Shrinkage Control}

A series of experiments were carried out to reveal the influence of the applied voltage, solution concentration, distance between electrodes, and rotation speed of the collector on the shrinkage of the TPU (MM5520) membrane in the rotational direction and transverse direction (of the roller). The solution flow rate was kept at $1.2 \mathrm{~mL} / \mathrm{h}$ in these experiments.

Figure 5I presents the shrinkage ratio in the rotational direction versus that in the transverse direction of all experimental results, which are divided into four groups ((a), (b), (c) and (d)). Refer to the legend for the processing parameters (parameters are labeled in the sequence of applied voltage $(\mathrm{kV})$, rotation speed of the collector $(\mathrm{rpm} / \mathrm{min})$, solution concentration $(\mathrm{wt} \%)$, and distance between electrodes $(\mathrm{cm}))$ of the samples applied in each test. Each test includes at least five samples, which were cut out from the middle part of the same piece of the as-fabricated membrane. Refer to Table S1 in the Supplementary Materials for the processing parameters of each group and the shrinkage ratios of each sample in both the rotational and transverse directions. 


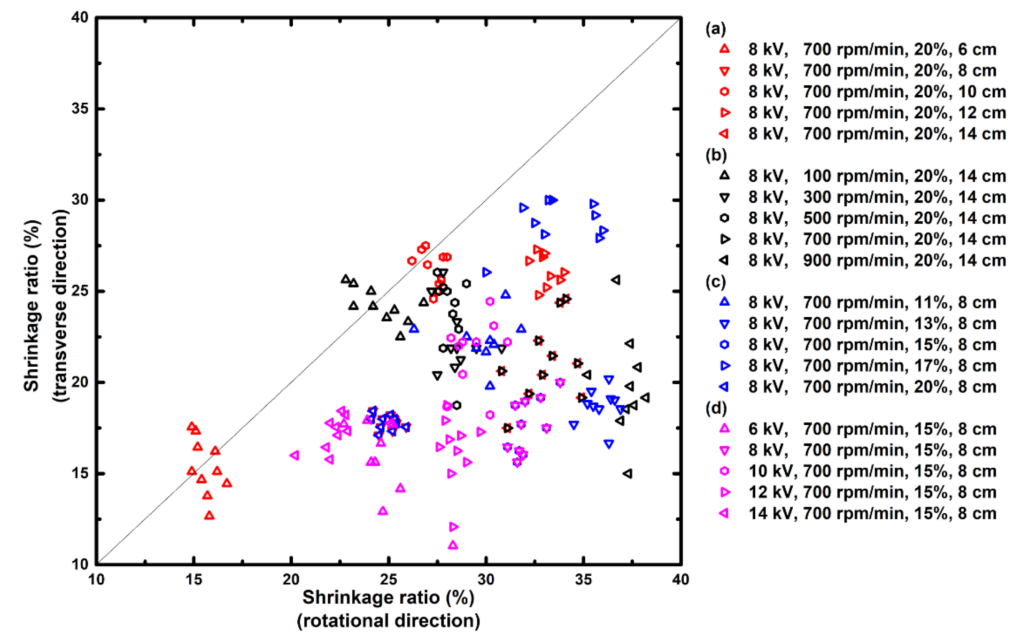

(I)
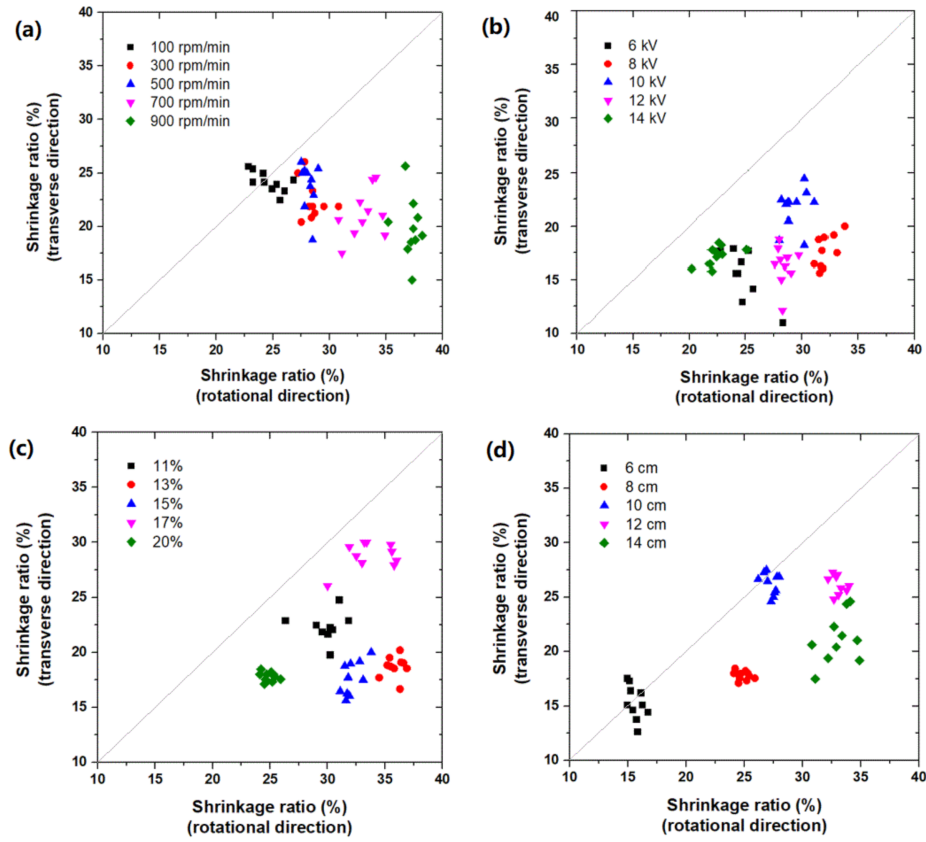

(II)

Figure 5. Shrinkage ratio along the rotational direction versus shrinkage ratio along the transverse direction (solution flow rate: $1.2 \mathrm{~mL} / \mathrm{h}$ ). (I) Overlapping of all experimental results; (II) results of parametric study. (a) Rotation speed of collector; (b) applied voltage; (c) solvent concentration; (d) distance between electrodes.

Although this parametric investigation is far away from complete, it is found that most of the results are distributed on the lower right of the diagonal, that is, the shrinkage ratio in the rotational direction is greater than that in the transverse direction. This is apparently due to the drafting and alignment effect of the roller drum.

All samples are able to shrink over $10 \%$ in both the rotational and transverse directions. The maximum shrinkage in the rotational direction is over $37.5 \%$, while that in the transverse direction is slightly less, about $30 \%$. Below the diagonal, the obtained experimental results cover almost the whole area of 15\% to 35\% (in the rotational direction) and 15\% to $30 \%$ (in the transverse direction).

Some results of different groups overlap. Hence, there might be multiple ways to achieve the required shrinkage ratios in both directions.

In Figure 5II, we plot the results of each group separately to reveal the influence of each parameter. Refer to Figure S6 in the Supplementary Materials for the relationship 
between the average shrinkage ratios in both directions as a function of the parameter that varies in that group of tests.

In Figure 5(IIa), the influence of the rotation speed of the roller is apparent and more or less monotonic. In particular, when the rotation speed is over $500 \mathrm{rpm}$, the shrinkage ratios change rapidly (refer to Figure S6a in the Supplementary Materials). A higher roller drum speed tends to align fibers better into the direction of rotation (refer to Figures S4 and S5 in the Supplementary Materials) and may cause higher pre-strain in the fibers. Consequently, the shrinkage ratio in the direction of rotation increases with the increase of the rotation speed of the roller, while the shrinkage ratio in the transverse direction reduces but in a lower level (Figure 5a, Figure S6a in the Supplementary Materials). Therefore, the shrinkage ratios in the rotational direction and the transverse direction can be tailored by varying the rotation speed of the roller drum, even in a layer-by-layer manner in the process of membrane fabrication to result in a gradient 2D shrinkage ratio along the thickness direction of the membrane.

Since $700 \mathrm{rpm} / \mathrm{min}$ was the applied rotation speed in all other groups, it is expected that the results of those tests should be all underneath the diagonal.

Figure 5(IIa) also shows that with the increase in the rotation speed, the results spread over a wider range. For example, the shrinkage ratio in the transverse direction of $900 \mathrm{rpm} / \mathrm{min}$ is from about $15 \%$ to $25 \%$.

In Figure 5(IIb), the variable is the applied voltage. According to Figure S6b in the Supplementary Materials, for this particular set of fixed parameters, there is no clear trend for the influence of the applied voltage. This is also found for the influence of the solution concentration in Figure 5(IIc) and Figure S6c in the Supplementary Materials. The applied voltage and solution concentration affect the shrinkage ratio of the membranes through the competition among a number of factors, such as flight time, stretching force, solvent evaporation rate, and solution viscosity. It is interesting to point out that in Figure 5(IIc), the results of $20 \%$ solution concentration are within a very small area, which implies that this membrane is very uniform and high quality.

The electric field strength and the flight distance of the fibers vary according to the distance from the nozzle to the collector. In Figure 5(IId), the variable is the distance between electrodes. By varying the distance from 6 to $12 \mathrm{~cm}$, the shrinkage ratio in the rotational direction increases from about $15 \%$ to $35 \%$ in an approximately linear manner (refer to Figure S6d in the Supplementary Materials). Further increase in the distance to $14 \mathrm{~cm}$ does not increase the shrinkage ratio but causes wider spreading of the results and decrease in the shrinkage ratio in the transverse direction. Since the influence of the distance on the shrinkage ratio in the transverse direction is not monotonic, the results of five different distances do not follow any clear trend. The results of some groups (namely $8 \mathrm{~cm}, 10 \mathrm{~cm}$, and $12 \mathrm{~cm}$ ) are within a small area, which indicates that the change distance is a more effective way to result in uniform and high-quality membranes.

A surprise spotted in Figure 5(IId) is that the results of two distances, namely $6 \mathrm{~cm}$ and $10 \mathrm{~cm}$, are around the diagonal, although the applied rotation speed is $700 \mathrm{rpm} / \mathrm{min}$, while a distance of $8 \mathrm{~cm}$, which is in between, is far away from the diagonal but within a small area.

Although the results reported here are limited, the following findings are apparent:

- There are many processing parameters that affect the shrinkage ratio of the electrospun membranes. Environmental conditions, such as room temperature and humidity, and solution flow rate are not included in this study.

- There are different ways to tailor the shrinkage ratios in both directions.

- Some of the ways are more effective to result in uniform and high-quality membranes.

\section{Conclusions}

In this paper, a gradient pre-strain field is identified as the underlying mechanism for the shrinkage of the electrospun membranes, which is activated by the stimulusresponsive shape memory effect. Crimped fibers in the elecrospun membranes after 
shrinkage (revealed by SEM) provide solid evidence for buckling, which is associated with the gradient pre-strain field in the cross-section of the fibers. Depending on the electrospun process and material, there are two types of buckling conditions. Due to the nature of the electrospun membrane, the out-shell of the resulted polymeric fibers are pre-stretched, while the inner core is without stretching or less stretched. Hence, shrinkage is more or less an intrinsic feature of all electrospun membranes. Many processing parameters can affect the gradient pre-strain field and therefore the shrinkage ratio of the membranes.

A series of experiments were carried out to reveal the influence of the applied voltage, solution concentration, distance between electrodes, and rotation speed of collector on the shrinkage ratios of a commercial TPU in the rotational direction of the roller and the transverse direction. Although this parametric investigation is far away from complete, it is concluded that there are different ways to achieve the required shrinkage ratios in both directions. Some of them might be more effective to achieve good uniformity.

Via optimization of these parameters, it is possible not only to control the shrinkage but also to realize gradient shrinkage in two in-plane directions along the thickness direction of the membranes.

Supplementary Materials: The following are available online at https:/ /www.mdpi.com/article/10 .3390/mi12080920/s1. Figure S1: Typical SEM images of TPU MM5520 electrospun nanofiber membrane before (a1 and a2) and after (b1 and b2) ethanol infiltration. Figure S2: (a) Photographs of TPU electrospun membranes before (a1) and after (b1) heating to $70^{\circ} \mathrm{C}$. (b) SEM images of the electrospun nanofiber membrane before (a2) and after (b2) the shrinkage. Figure S3: (a) Photographs of poly (lactic acid) (PLA) electrospun membranes before (a1) and after (b1) ethanol infiltration. (b) SEM images of the electrospun nanofiber membrane before (a2) and after (b2) the shrinkage. Figure S4: SEM micrographs of TPU electrospun nanofiber membrane produced at different rotation speeds of the roller collector. Figure S5: Fast Fourier transform (FFT) analysis of Figure S4a,b,d. Directionality plugin of ImageJ software (http:/ / fiji.sc/Fiji (accessed on 1 April 2021), Ashburn, VA, USA) was used to analyze the alignment of fiber. Figure S6: Average shrinkage ratio of electrospun nanofibers membrane produced using different electrospinning parameters. Video S1: Controlled ethanol wetting of membrane. Table S1: Processing parameters and experimental results of shrinkage ratios.

Author Contributions: Conceptualization, F.F., H.W. (Huaquan Wang), W.M.H.; Investigation, F.F., H.W. (Huaquan Wang) and Y.C.; Supervision, W.M.H., H.W. (Han Wang); Writing-original draft, F.F.; Writing-review and editing, W.M.H.; N.C., X.C. (Xindu Chen) and X.C. (Xin Chen) supervised and offered various suggestions to improve this manuscript. All authors have read and agreed to the published version of the manuscript.

Funding: This work was financially supported by Project of Jihua Laboratory (No.X190071UZ190), Foshan Science and Technology Innovation Project (No.2020001004407), the Science and Technology Program of Guangzhou, China (No. 202102010251).

Conflicts of Interest: The authors declare no conflict of interest.

\section{References}

1. Wang, Z.; Zhang, W.; Xie, C.; Wen, F.; Ma, C.; Lin, N.; Thian, E.S.; Wang, X. Geometric anisotropy on biomaterials surface for vascular scaffold design: Engineering and biological advances. J. Phys. Mater. 2019, 2, 032003. [CrossRef]

2. Jiang, J.; Shao, Z.; Wang, X.; Zhu, P.; Deng, S.; Li, W.; Zheng, G. Three-dimensional composite electrospun nanofibrous membrane by multi-jet electrospinning with sheath gas for high-efficiency antibiosis air filtration. Nanotechnology 2021, 32, 245707. [CrossRef]

3. Yu, L.; Zhou, P.; Wu, D.; Wang, L.; Lin, L.; Sun, D. Shoepad nanogenerator based on electrospun PVDF nanofibers. Microsyst. Technol. 2018, 25, 3151-3156. [CrossRef]

4. Ru, C.; Wang, F.; Pang, M.; Sun, L.; Chen, R.; Sun, Y. Suspended, Shrinkage-Free, Electrospun PLGA Nanofibrous Scaffold for Skin Tissue Engineering. ACS Appl. Mater. Interfaces 2015, 7, 10872-10877. [CrossRef]

5. Apsite, I.; Stoychev, G.; Zhang, W.; Jehnichen, D.; Xie, J.; Ionov, L. Porous Stimuli-Responsive Self-Folding Electrospun Mats for 4D Biofabrication. Biomacromolecules 2017, 18, 3178-3184. [CrossRef] [PubMed]

6. Fang, F.; Wang, H.; Wang, H.; Gu, X.; Zeng, J.; Wang, Z.; Chen, X.; Chen, X.; Chen, M. Stretchable MXene/Thermoplastic Polyurethanes based Strain Sensor Fabricated Using a Combined Electrospinning and Electrostatic Spray Deposition Technique. Micromachines 2021, 12, 252. [CrossRef] 
7. Xie, Z.; Buschle-Diller, G.; DeInnocentes, P.; Bird, R.C. Electrospun poly(D,L)-lactide nonwoven mats for biomedical application: Surface area shrinkage and surface entrapment. J. Appl. Polym. Sci. 2011, 122, 1219-1225. [CrossRef]

8. Xie, Z.; Buschle-Diller, G. Electrospun poly(D,L-lactide) fibers for drug delivery: The influence of cosolvent and the mechanism of drug release. J. Appl. Polym. Sci. 2010, 115, 1-8. [CrossRef]

9. Wang, J.; Windbergs, M. Influence of polymer composition and drug loading procedure on dual drug release from PLGA:PEG electrospun fibers. Eur. J. Pharm. Sci. 2018, 124, 71-79. [CrossRef]

10. Rianjanu, A.; Kusumaatmaja, A.; Suyono, E.A.; Triyana, K. Solvent vapor treatment improves mechanical strength of electrospun polyvinyl alcohol nanofibers. Heliyon 2018, 4, e00592. [CrossRef]

11. Rozent, O.; Beilin, V.V.; Shter, G.E.; Grader, G.S.; Jones, J. Deformation Control During Thermal Treatment of Electrospun PbZr0.52Ti0.48O3 Nanofiber Mats. J. Am. Ceram. Soc. 2016, 99, 1550-1556. [CrossRef]

12. Zhang, L.; Liu, L.-G.; Pan, F.-L.; Wang, D.-F.; Pan, Z.-J. Effects of Heat Treatment on the Morphology and Performance of PSU Electrospun Nanofibrous Membrane. J. Eng. Fibers Fabr. 2012, 7, 1558. [CrossRef]

13. Zhao, Y.; Miao, X.; Lin, J.; Li, X.; Bian, F.; Wang, J.; Zhang, X.; Yue, B. Coiled Plant Tendril Bioinspired Fabrication of Helical Porous Microfibers for Crude Oil Cleanup. Glob. Chall. 2017, 1, 1600021. [CrossRef] [PubMed]

14. Wu, H.; Zheng, Y.; Zeng, Y. Fabrication of Helical Nanofibers via Co-Electrospinning. Ind. Eng. Chem. Res. 2015, 54, 987-993. [CrossRef]

15. Silva, P.E.S.; Vistulo de Abreu, F.; Godinho, M.H. Shaping helical electrospun filaments: A review. Soft Matter 2017, 13, 6678-6688. [CrossRef]

16. Otsuka, K.; Wayman, C.M. (Eds.) Shape Memory Materials; Cambridge University Press: Cambridge, UK, 1998.

17. Huang, W.M.; Ding, Z.; Wang, C.C.; Wei, J.; Zhao, Y.; Purnawali, H. Shape memory materials. Mater. Today 2010, 13 , 54-61. [CrossRef]

18. Huang, W.M.; Zhao, Y.; Wang, C.C.; Ding, Z.; Purnawali, H.; Tang, C.; Zhang, J.L. Thermo/chemo-responsive shape memory effect in polymers: A sketch of working mechanisms, fundamentals and optimization. J. Polym. Res. 2012, 19, 1-34. [CrossRef]

19. Huang, W.M.; Yang, B.; An, L.; Li, C.; Chan, Y.S. Water-driven programmable polyurethane shape memory polymer: Demonstration and mechanism. Appl. Phys. Lett. 2005, 86, 114105. [CrossRef]

20. Lu, H.B.; Huang, W.M.; Yao, Y.T. Review of chemo-responsive shape change/memory polymers. Pigment Resin Technol. 2013, 42, 237-246. [CrossRef]

21. Salvekar, A.V.; Huang, W.M.; Xiao, R.; Wong, Y.S.; Venkatraman, S.S.; Tay, K.H.; Shen, Z.X. Water-responsive shape recovery induced buckling in biodegradable photo-cross-linked poly (ethylene glycol)(PEG) hydrogel. Acc. Chem. Res. 2017, 50, 141-150. [CrossRef]

22. Fan, K.; Huang, W.M.; Wang, C.C.; Ding, Z.; Zhao, Y.; Purnawali, H.; Liew, K.C.; Zheng, L.X. Water-responsive shape memory hybrid: Design concept and demonstration. Express Polym. Lett. 2011, 5, 409-416. [CrossRef]

23. Wu, X.L.; Huang, W.M.; Lu, H.B.; Wang, C.C.; Cui, H.P. Characterization of polymeric shape memory materials. J. Polym. Eng. 2017, 37, 1-20. [CrossRef]

24. Ding, Z.; Yuan, C.; Peng, X.; Wang, T.; Qi, H.J.; Dunn, M.L. Direct 4D printing via active composite materials. Sci. Adv. 2017, 3, e1602890. [CrossRef]

25. Wang, C.C.; Zhao, Y.; Purnawali, H.; Huang, W.M.; Sun, L. Chemically induced morphing in polyurethane shape memory polymer micro fibers/springs. React. Funct. Polym. 2012, 72, 757-764. [CrossRef]

26. Yang, B.; Huang, W.M.; Li, C.; Li, L. Effects of moisture on the thermomechanical properties of a polyurethane shape memory polymer. Polymer 2006, 47, 1348-1356. [CrossRef]

27. Taylor, G.I.; Van Dyke, M.D. Electrically driven jets. Proc. R. Soc. Lond. A 1969, 313, 453-475. [CrossRef]

28. Yarin, A.L.; Koombhongse, S.; Reneker, D.H. Bending instability in electrospinning of nanofibers. J. Appl. Phys. 2001, 89, 3018-3026. [CrossRef]

29. Deshawar, D.; Gupta, K.; Chokshi, P. Electrospinning of polymer solutions: An analysis of instability in a thinning jet with solvent evaporation. Polymer 2020, 202, 122656. [CrossRef]

30. Wu, X.-F.; Salkovskiy, Y.; Dzenis, Y.A. Modeling of solvent evaporation from polymer jets in electrospinning. Appl. Phys. Lett. 2011, 98, 223108. [CrossRef]

31. Sun, L.; Huang, W.M. Mechanisms of the multi-shape memory effect and temperature memory effect in shape memory polymers. Soft Matter 2010, 6, 4403-4406. [CrossRef]

32. Zhao, Y.; Chun Wang, C.; Min Huang, W.; Purnawali, H. Buckling of poly(methyl methacrylate) in stimulus-responsive shape recovery. Appl. Phys. Lett. 2011, 99, 131911. [CrossRef]

33. Huang, W.M.; Yang, B.; Fu, Y.Q. Polyurethane Shape Memory Polymers; CRC Press: New York, NY, USA, 2011. 